Research Paper

\title{
Evaluating Potential of Electromagnetic Microwaves on Destruction
} Acanthamoeba Cysts

\author{
Zahra Eslamirad $^{1} \odot$, Reza Hajihossein ${ }^{1} \odot{ }^{*}$ Homa Soleimani $^{2}$
}

1. Department of Parasitology and Mycology, School of Medicine, Arak University of Medical Sciences, Arak, Iran

2. Department of Medical Physics and Physiology, School of Medicine, Arak University of Medical Sciences, Arak, Iran.

\begin{tabular}{c|l}
$\begin{array}{c}\text { Use your device to scan } \\
\text { and read the article online }\end{array}$ \\
Citation: Eslamirad Z, Hajihossein R, Soleimani H. [Evaluating Potential of Electromagnetic Microwaves on Destruction Acan- \\
thamoeba Cysts (Persian)]. Complementary Medicine Journal. 2020; 9(4):3868-3877. https://doi.org/10.32598/cmja.9.4.583.3
\end{tabular}

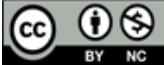

Article Info:

Received: 08 Nov 2019

Accepted: 18 Dec 2019

Available Online: 01 Mar 2020

Keywords:

Acanthamoeba

Free-living Amoeba,

Microwave, Electro-

magnetic waves

\section{ABSTRACT}

Objective Acanthamoeba is a free-living and opportunistic amoeba that the potential of this parasite to convert to a cyst, making its treatment difficult. In this study, we investigated the effect of microwave radiation on Acanthamoeba cysts in vitro.

Methods Acanthamoeba cysts were reproduced in the culture medium. We divided 16 test tubes containing cysts into two groups. The contents of 8 tubes were irradiated with continuous microwave in the time range of $0-120 \mathrm{~s}$, and the next 8 tubes were irradiated with repetitive microwave in the range of 0-360 s. The mortality of cysts at the end of irradiation was recorded and compared with control.

Results Continuous and repetitive irradiation of microwave resulted in mortality of 100 (\%) of Acanthamoeba cysts during 120 and 360 seconds, respectively. In continuous mode, parasite mortality in all groups was significantly different from the control group $(P<0.0001)$. But in the repetition pattern only in groups that had been irradiated for more than 60 seconds, parasite mortality was significantly different from the control group $(\mathrm{P}<0.0001)$.

Conclusion The change of radiation mode from continuous to repetitive, reduces the thermal effects of the microwaves but does not affect the mortality rate of Acanthamoeba cysts. Therefore, probably the impact of microwaves was not only caused by the increase in ambient heat but also its another property is involved in the death of Acanthamoeba.

\section{Extended Abstract}

\section{Introduction}

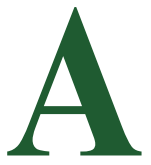

canthamoeba is a free-living amoeba that plenty of them live around us [1]. The high prevalence of this parasite increases the risk of exposure to humans. Because the parasite is opportunistic, under appropriate conditions, human health at risk and caused acanthamoe- biasis disease. The neurological disorders caused by this parasite often lead to death and the treatment of visual disorders has not been effective so far [2] because the parasite in vitro and in vivo environments become highly resistant cysts and hard to be treated and controlled [3].

Although a wide range of chemical drugs can be used to treat the disease, they may have side effects $[5,6]$. Therefore, the searches for an alternative natural and traditional medicine rather than chemical drugs continue [2].

* Corresponding Author:

Homa Soleimani, PhD.

Address: Department of Medical Physics and Physiology, School of Medicine, Arak University of Medical Sciences, Arak, Iran.

Tel: +98 (86) 34173521

E-mail: dr.hsoleimani@arakmu.ac.ir 
Recently the effect of some herbal compounds in the treatment of this disease has been studied and confirmed. Since human beings are exposed to high-frequency waves through natural and artificial sources, the effects of these waves on the control or treatments of microorganisms including parasites have also been addressed in some studies $[8,9]$. In this study, we investigated the effect of microwave radiation on Acanthamoeba cysts in vitro.

\section{Materials and Methods}

An experimental study was performed on Acanthamoeba cysts in vitro. The parasitic cysts were cultured and a parasitic suspension containing 19000 to 20,000 cysts per $\mathrm{ml}$ was prepared. Sixteen test tubes containing $100 \mu \mathrm{l}$ of parasitic suspension were prepared and divided into two groups including 8 tubes [16]. These tubes were irradiated by microwaves oven with a power of 1550 watts and a frequency of $2450 \mathrm{MHz}$. The contents of 8 tubes were irradiated with continuous microwave in $0-120 \mathrm{~s}$ time interval (first group) and the next 8 tubes were irradiated with re- petitive microwave in 0-360 s time interval (second group). In both groups, the first tube with zero radiation time was considered as the control. The mortality of cysts at the end of irradiation was recorded and compared with control. The temperature of the parasitic suspension was recorded before and after the irradiation and the difference between the initial and final temperature of the experiment was recorded as $\Delta \mathrm{T}$. Each experiment was repeated 3 times.

\section{Results}

The results of this study showed that continuous and repetitive microwave irradiation resulted in $100 \%$ death of the parasite cysts in 120 and $360 \mathrm{~s}$, respectively (Table 1 and 2). ANOVA analysis between the rate of the parasite after continuous microwave irradiation indicates that the parasitic mortality rate in the control group was significantly different from other irradiation times (except $10 \mathrm{~s})(\mathrm{P}<0.0001)$. Statistical analysis also showed that parasite mortality rates were significantly different between all continuous irradiation times (except for 30 and $90 \mathrm{~s})(\mathrm{P}<0.0001)$.

Table 1. The mortality rate of Acanthamoeba cysts after exposure to continuous microwave irradiation (first group)

\begin{tabular}{cccc}
\hline \multirow{2}{*}{ Subgroup } & Continuous Irradiation Time $(\mathbf{s})$ & Mean \pm SD & Mortality (\%) \\
\cline { 3 - 4 } & & $\left(\mathbf{C}^{\circ}\right) \mathbf{~} \mathbf{T}$ & $0 \pm 0$ \\
\hline 1 & 10 & $0 \pm 0$ & $0 \pm 0$ \\
\hline 2 & 30 & $8.1 \pm 0.3$ & $2.7 \pm 0.6$ \\
\hline 4 & 45 & $24 \pm 0.6$ & $2.7 \pm 0.6$ \\
\hline 5 & 60 & $44.6 \pm 1.4$ & $3 \pm 1$ \\
\hline 7 & 90 & $45.9 \pm 1.4$ & $3 \pm 0$ \\
\hline 8 & 105 & $69.3 \pm 4.5$ & $5.7 \pm 0.58$ \\
& 120 & $83.3 \pm 5.5$ & $100 \pm 0$ \\
\hline
\end{tabular}

Table 2. The mortality rate of Acanthamoeba cysts after exposure to repetitive microwave irradiation (second group)

\begin{tabular}{|c|c|c|c|}
\hline \multirow{2}{*}{ Subgroup } & \multirow{2}{*}{ Repetitive Irradiation Time $(\mathbf{s}) \times$ Times } & \multicolumn{2}{|c|}{ Mean $\pm S D$} \\
\hline & & $\left(C^{\circ}\right) \Delta T$ & Mortality (\%) \\
\hline 1 & 0 & $0 \pm 0$ & $0 \pm 0$ \\
\hline 2 & $1 \times 10$ & $8.2 \pm 0.7$ & $0 \pm 0$ \\
\hline 3 & $3 \times 10$ & $8.2 \pm 1.1$ & $0 \pm 0$ \\
\hline 4 & $6 \times 10$ & $8.7 \pm 1$ & $1 \pm 1$ \\
\hline 5 & $6 \times 20$ & $16.2 \pm 0.7$ & $1.3 \pm 0.58$ \\
\hline 6 & $6 \times 30$ & $24.8 \pm 0.3$ & $3.3 \pm 0.58$ \\
\hline 7 & $6 \times 45$ & $42.1 \pm 0.26$ & $5.3 \pm 1.15$ \\
\hline 8 & $6 \times 60$ & $46.9 \pm 1.55$ & $100 \pm 0$ \\
\hline
\end{tabular}


ANOVA analysis between the rate of the parasite after repetitive microwave irradiation indicates that the parasitic mortality rate in the control group was significantly different from other irradiation times (except 10, 30, and $60 \mathrm{~s}$ ) $(\mathrm{P}<0.0001)$.

\section{Discussion}

Acanthamoeba is a pathogenic organism that produces resistant cysts. These cysts lead to the ineffectiveness of conventional drugs against this parasite. Cysts of this parasite are resistant to chemical drugs or require overdosage of drugs to eliminate cysts. So finding and using drugs of natural origin or other treatments that destroy the cyst wall or wall synthesis obstacle have been the focus of researchers [17].

Also, the use of therapeutic properties of mechanical factors such as sound waves has been considered in recent years. So that some researchers use the waves (mechanical or electromagnetic) to eliminate pathogenic organisms and treat diseases. One of the first medical applications of electromagnetic waves has been to destroy cancer cells [20]. In recent years the effects of High-intensity Focused Ultrasound (HIFU) and microwave on some parasitic agents have been proven $[8,9,15]$.

In this study, the effect of microwave as a high-frequency non-ionizing radiation with thermal effects on highly resistant Acanthamoeba cysts was investigated. The results showed that the effect of microwave irradiation on these cysts depends on the duration of irradiation. On the other hand, the mortality rate of Acanthamoeba parasites in continuous and repetitive microwave irradiation did not exceed $6 \%$ during 105 and $270 \mathrm{~s}$, respectively, but when the irradiation time increased to 120 and $360 \mathrm{~s}$ respectively, the mortality rate rapidly increased to $100 \%$.

The results of the study showed that during continuous irradiation of the microwave for $105 \mathrm{~s}$, the mortality rate of Acanthamoeba cysts was only $5.7 \%$, and $\Delta \mathrm{T}$ about $83^{\circ} \mathrm{C}$. However, during the repetitive microwave irradiation for $270 \mathrm{~s}$, the $\Delta \mathrm{T}$ was about $42^{\circ} \mathrm{C}$, and the mortality was almost similar to the continuous irradiation mode. According to these results, the change in the mode of microwave irradiation, from continuous to repetitive, resulted in a reduction of $\Delta \mathrm{T}$ but did not affect the mortality rate of Acanthamoeba cysts. Therefore, it seems that the effect of microwaves is not only caused by the increase in ambient heat but also another property of this type of wave is involved in the death of Acanthamoeba.

\section{Ethical Considerations}

\section{Compliance with ethical guidelines}

The project was approved by the Ethical Committee of Arak University of Medical Sciences (Code: 92-143-19).

Funding

This study extracted from a research project approved by the Arak University of Medical Sciences. We received financial support from the Deputy for Research and Technology of the university.

\section{Authors' contributions}

Conceptualization and Review \& editing: Zahra Eslamirad and Homa Soleimani; Investigation: Reza Hajihossein, Zahra Eslamirad, and Homa soleimani; Validation: Zahra Eslamirad; Supervision: Homa soleimani.

\section{Conflicts of interest}

The authors declared no conflict of interest.

\section{Acknowledgements}

The authors would like to thank the Research Deputy of Arak University of Medical Sciences for their financial support of this research (Number: 2348). 


\title{
ارزيابى يتانسيل امواج الكترومغناطيسى مايكرودر تخريب كيستهاى أكانتامبا
}

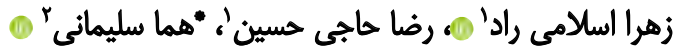

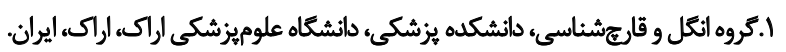

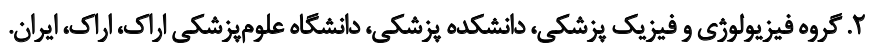

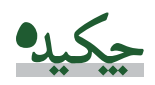

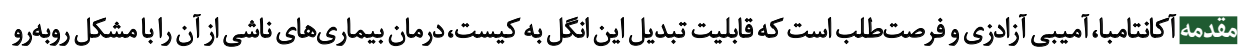

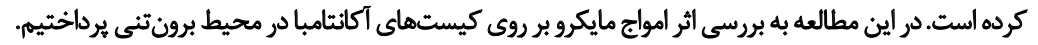

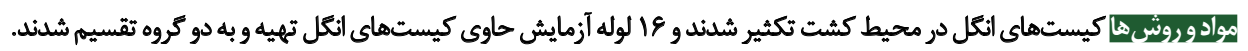

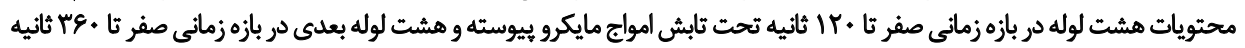

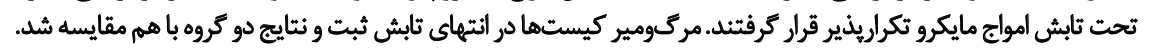

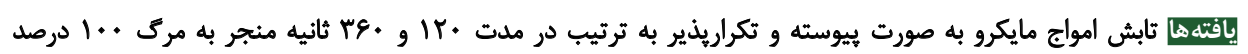

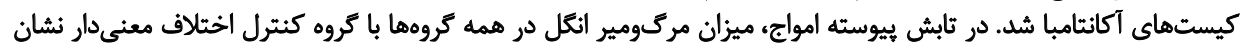

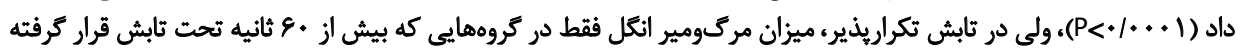

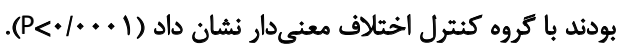

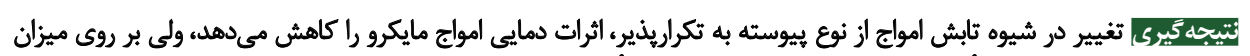

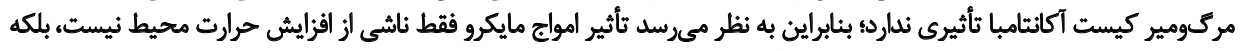

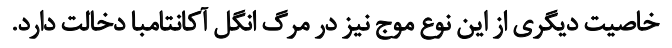

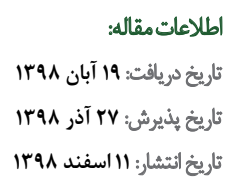

كليدواؤها:

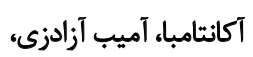

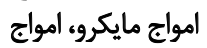

الكترومغناطيسى مانكي

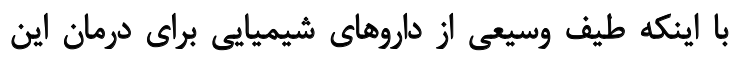

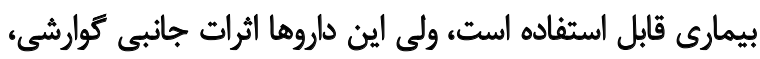

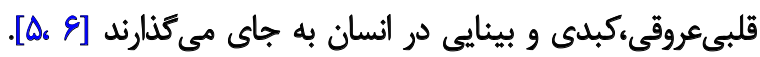

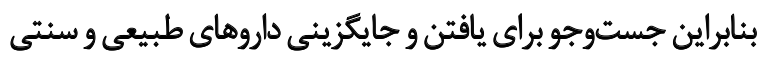

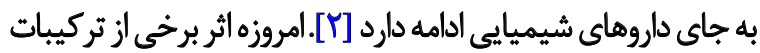

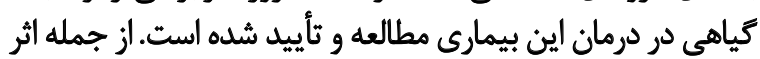

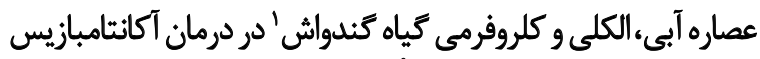

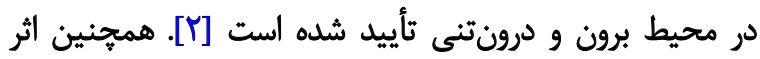

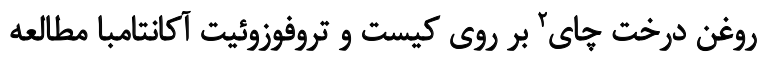

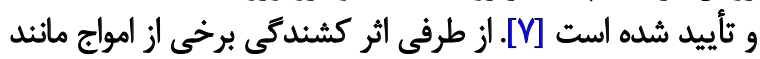

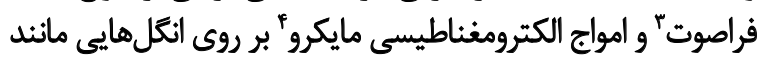

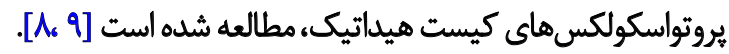

1. Artemisis annua $L$

2. Melaleuca alternifolia

3. HIFU

4. Microwave

dolito

آكانتامبا، آميبى آزادزى است كه بهفراوانى در محيط اطراف

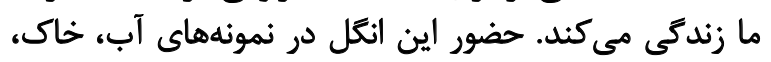

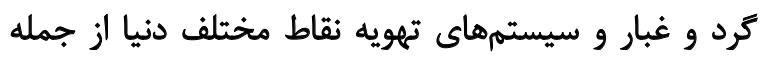

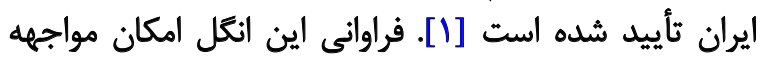

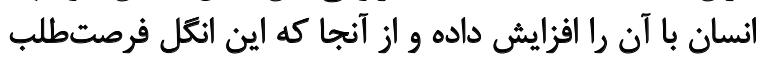

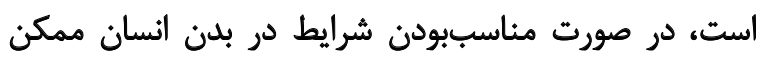

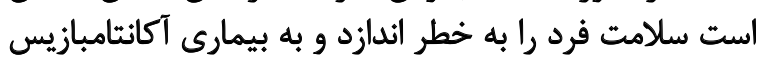

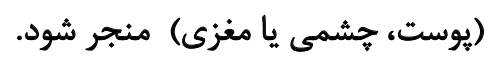

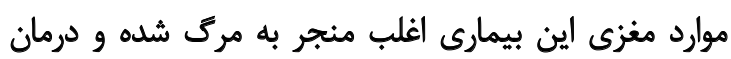

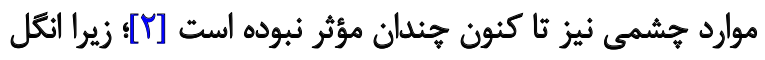

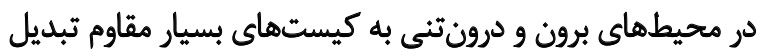

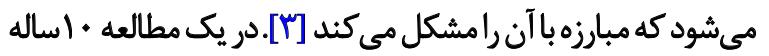

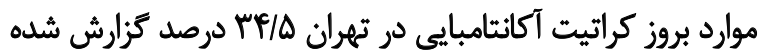

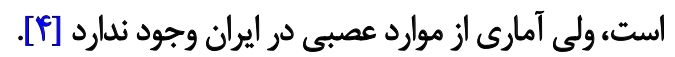

\section{ㅁ.}

: نويسئده مسئول:

دكتر هما سليمانى

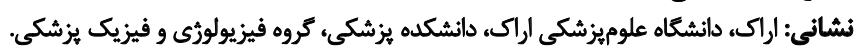
تلفن: يست الكترونيكي: dr.hsoleimani@arakmu.ac.ir 
سرم فيزيولوزى شستوشو و كيستها ازز روى آنها جمعآورى

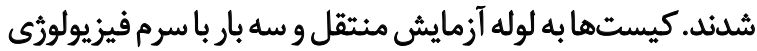

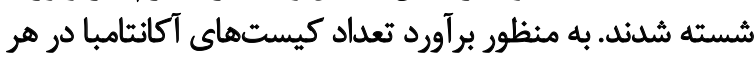

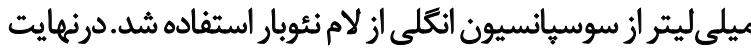

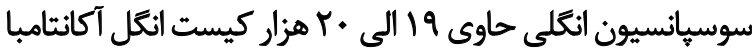

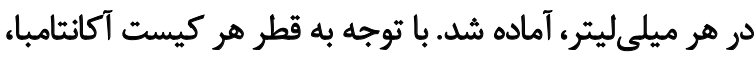

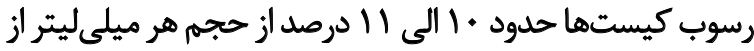

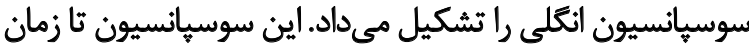

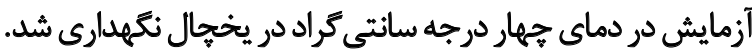

$$
\text { بروسى مركّومير انتمل }
$$

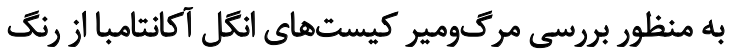

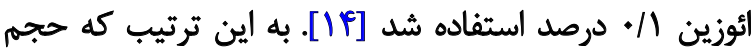

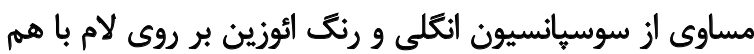

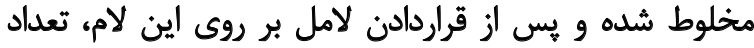

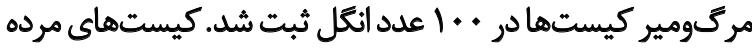

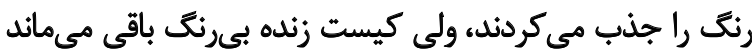

(تصوير شماره ()).

$$
\text { أزمايشها }
$$

اين مطالعه تجربى بر روى كيستهاى انغل آكائتامبادر محيط

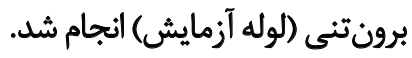

به منظور تابش امواج مايكرو از يك دستكاه اون سامسونك

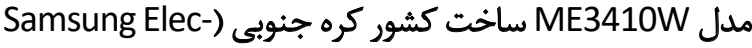
(tronic Co

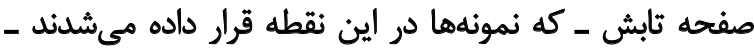

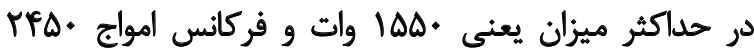

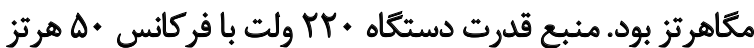

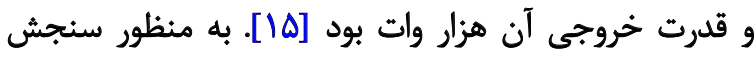

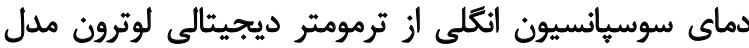

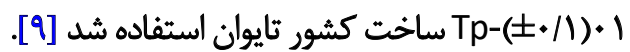

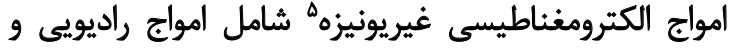

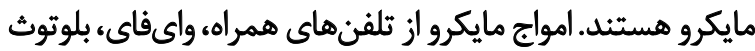

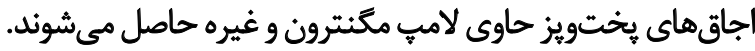
از آنجا كه افراد از طريق منابع طبيعى و مصنوعى (ساخته شده

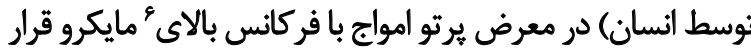

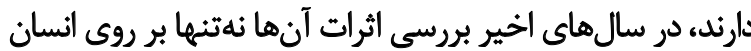

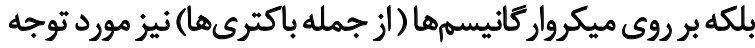

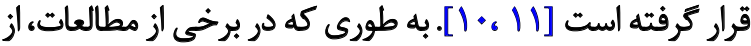

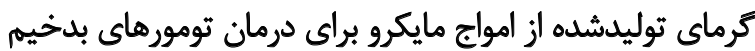

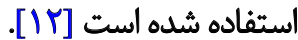

با توجه به اينكه سنجش يتانسيل عوامل در دسترسى

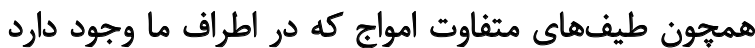

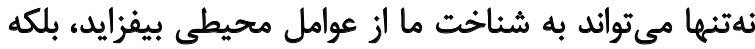

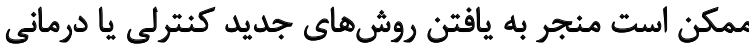

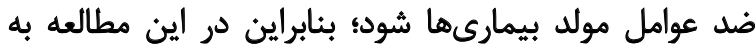

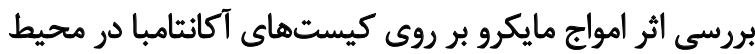
برونتنى برداخته شده است.

$$
\text { مواد و روشُها }
$$

\section{آمادهسازي كيستهاي آكائتامبا}

براي تهيه كيستهاى آكانتامبا، يكى از سويههاي بيمارىزاي

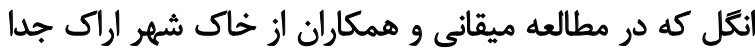

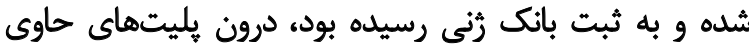

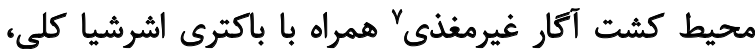

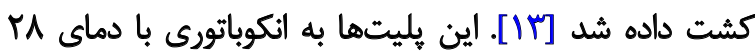

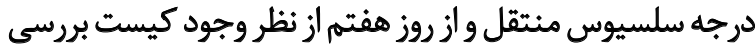

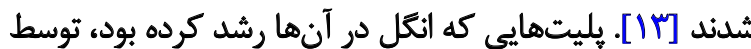

5. Radio frequency (RF)

6. High frequency

7. Non-nutrient Agar (NNA)

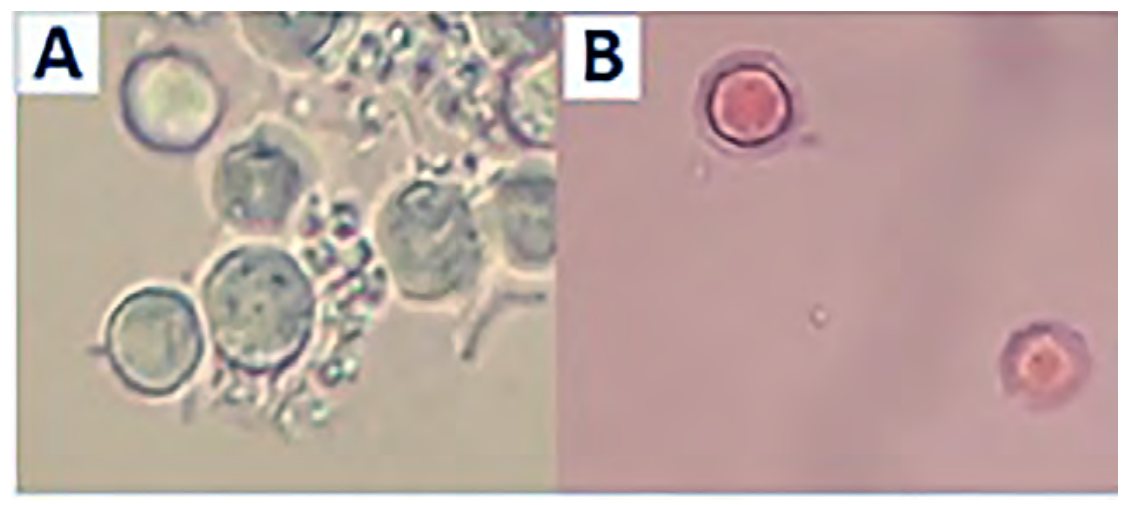


نشان داده شد. به منظور بررسى اختلاف بين كروهها با كروه

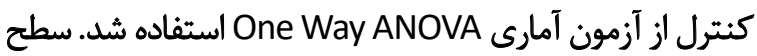

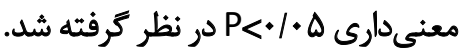

يافتهها

روند مركىومير كيستهاى آكانتامبا هـ از تابش امواج مايكرو

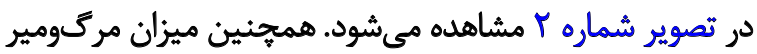

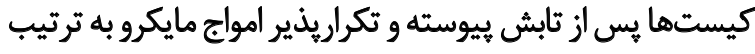

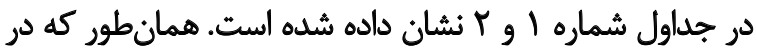

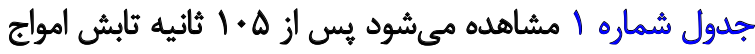

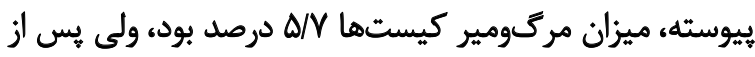

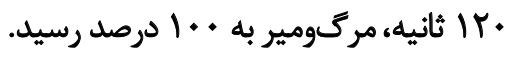

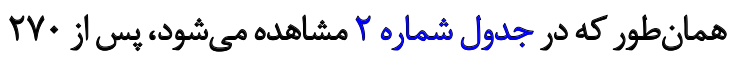

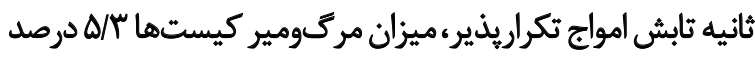

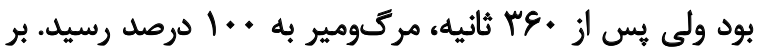

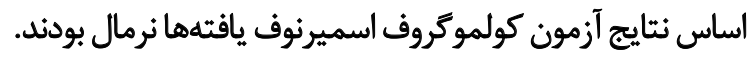

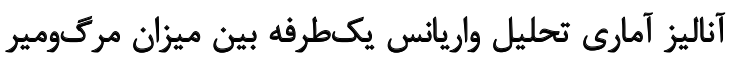

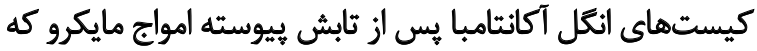

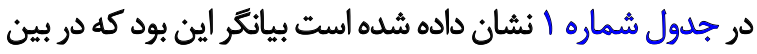

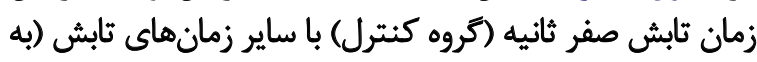

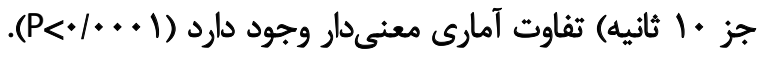

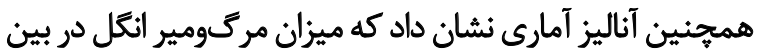

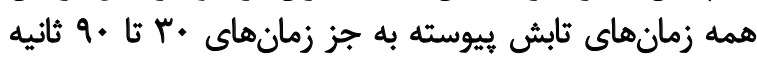

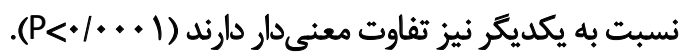
آناليز آمارى تحليل واريانس يكطرفه بين ميزان مركومير

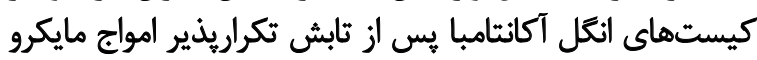

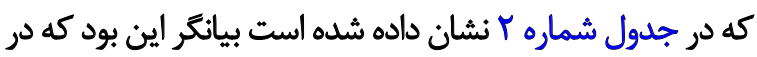

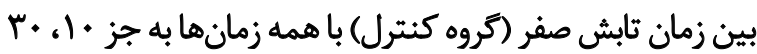

\section{مواجهه انتّل با ثابش بيوسته امواج مايكرو}

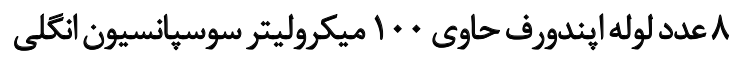

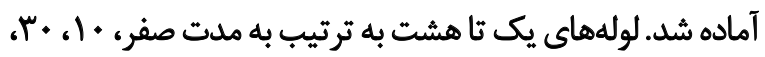

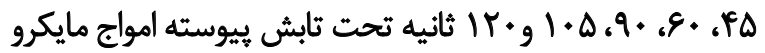

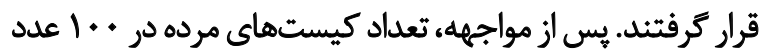

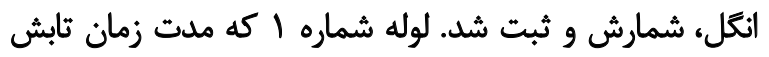

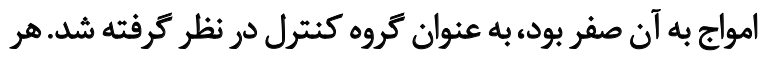

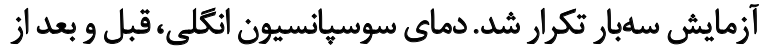
تابش امواج ثبت شد و تفاوت دماى ابتدايى و انتهايى آزمايش بـ به

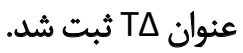
مواجها انئل با ثابش تكرارئذير امواج مايكرو

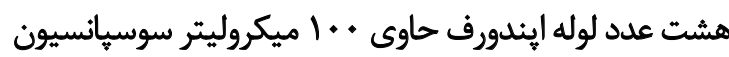

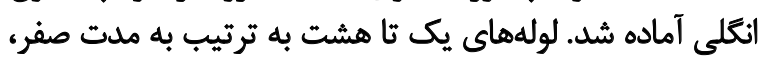

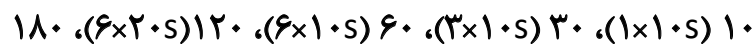
(\$)

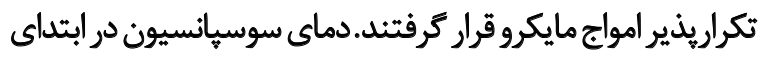

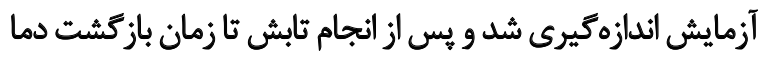

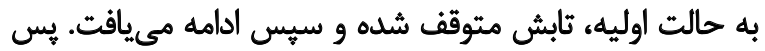

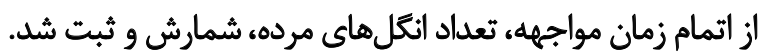

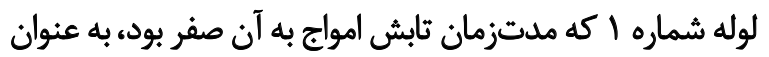
كروه كنترل در نظر كرفته شد. هر آزمايش سابهار تكرار شد.

\section{تجزيله و تحليل اطلاعات}

تجزيه و تحليل آمارى با استفاده از نرم افزار SPSS و نسخه

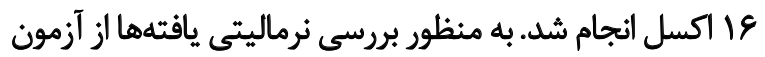

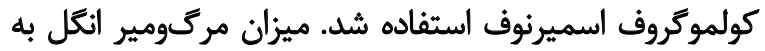

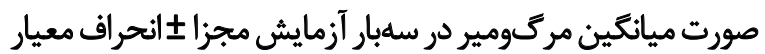

جدول ا. ميزان مركىومير كيستهاي آكانتامبا يس از ثابش يويوسته امواج مايكرو

\begin{tabular}{|c|c|c|c|}
\hline \multicolumn{2}{|c|}{ ميانكين $\mid$ انحراف معيار } & \multirow{2}{*}{ مدت زمان تابش (ييوسته) بر حسب ثانيه } & \multirow{2}{*}{ رديف } \\
\hline ميزان مركتومير (/) & $\left(C^{\circ}\right) \Delta T$ & & \\
\hline •. & \pm & • & 1 \\
\hline. \pm & $N \backslash \pm \cdot / r$ & 1. & r \\
\hline$T / N \pm \cdot / Q$ & $r q \pm . / q$ & r. & r \\
\hline$T / N \pm \cdot 18$ & $p q / \varepsilon \pm 1 / \varphi^{4}$ & PA & f \\
\hline$r \pm 1$ & $\varphi \Delta / q \pm 1 / \varphi$ & q. & $\Delta$ \\
\hline$r \pm$ & $s q r \pm \Psi / \Delta$ & q. & 8 \\
\hline$\Delta / V \pm \cdot / \Delta \Lambda$ & $\Delta \psi / \% \pm \Delta / \Delta$ & 1.0 & $v$ \\
\hline $1 . . \pm$ & $111 / r \pm \Delta / 1$ & ir. & $\wedge$ \\
\hline
\end{tabular}


جدول ז. ميزان مرك ومير كيستهاى آكائتامبا يس از تابش تكراريذير امواج مايكرو

\begin{tabular}{|c|c|c|c|}
\hline \multicolumn{2}{|c|}{ ميانكين||نحر افت معيار } & \multirow{2}{*}{ مدتزمان تابش بر حسب ثانيه × تعداد دقعات مواجهه } & \multirow{2}{*}{ رديف } \\
\hline 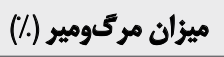 & $\left(C^{\circ}\right) \Delta T$ & & \\
\hline \pm & \pm & • & 1 \\
\hline \pm & $N T \pm \cdot N$ & $|x|$. & $r$ \\
\hline \pm & $N T \pm V / I$ & $r(x)$. & $r$ \\
\hline $\mid \pm 1$ & $N \vee \pm 1$ & $8 \times 1$. & r \\
\hline $1 / \% \pm+/ \Delta A$ & $\mid \& / r \pm \cdot N$ & sxy. & $\Delta$ \\
\hline$\Psi / \Psi \pm+/ \Delta A$ & $\Gamma \Psi / \wedge \pm . / \mu$ & sxy. & 8 \\
\hline$\Delta / T \pm V / \backslash \Delta$ & $R T / \Lambda \pm \cdot / R \varepsilon$ & sxita & $\checkmark$ \\
\hline $1 \ldots \pm$ & $f q / q \pm 1 / \Delta \Delta$ & $8 \times 9$. & $A$ \\
\hline
\end{tabular}

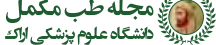

آكانتامباءاركانيسمى ياتورثن است كه مولد عفونتهاي شديدى

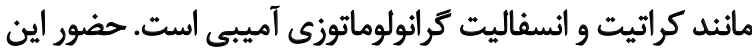

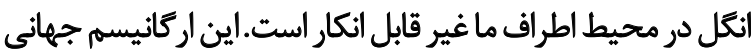

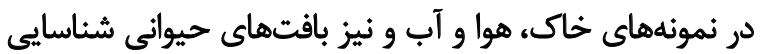

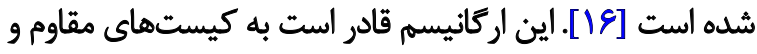

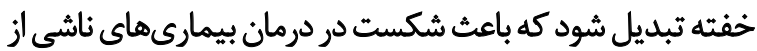

A1

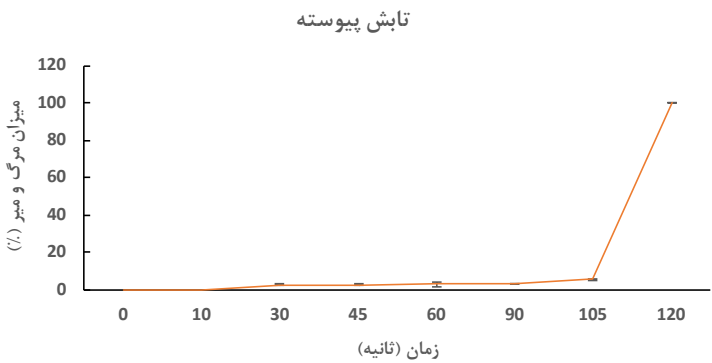

Mean of Mortality(\%)

B1

تابش تكراريذير

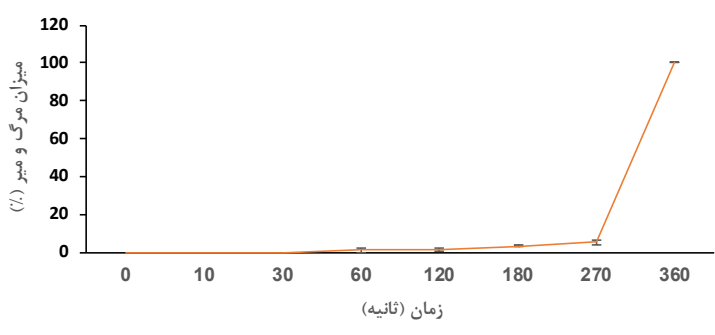

Mean of Mortality(\%)
و •9 ثانيه تفاوت معنى دار وجود دارد (1 . . P> P).

ب़

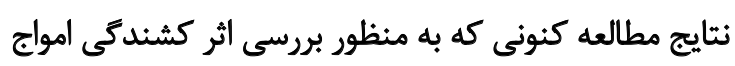

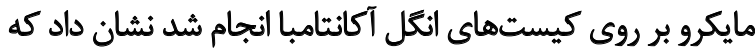
اثرات كشندكى اين امواج وابسته به مدتزمان تاني تابش امواج است.

A2

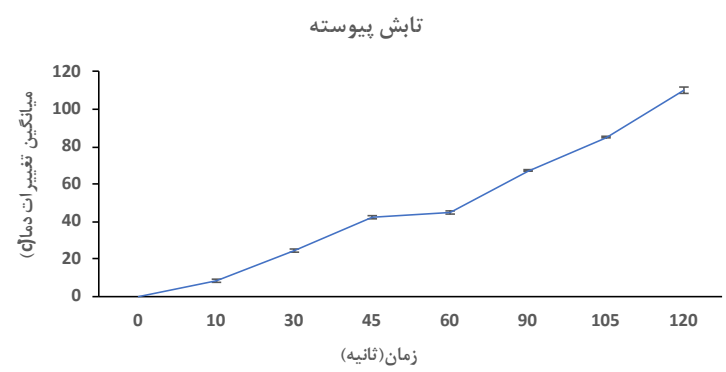

Mean of $\Delta \mathrm{T}$

B2

تابش تكراريذير

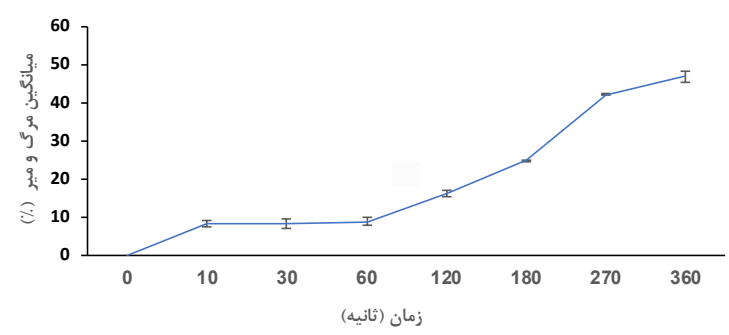

- Mean of $\Delta \mathrm{T}$

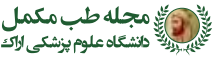

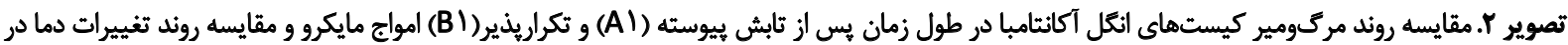

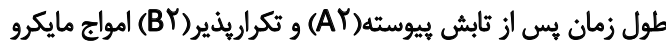


حاصل از اين امواج باعث تخريب لايه زاياى كيست و غيرفعال

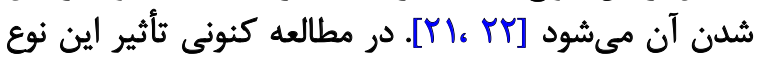

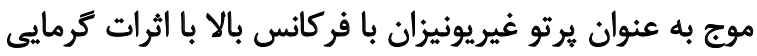

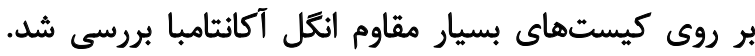

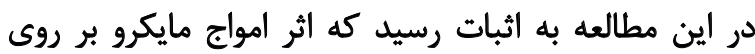

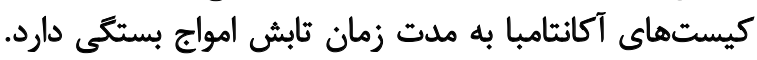

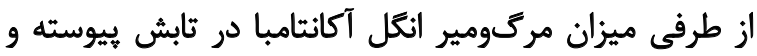

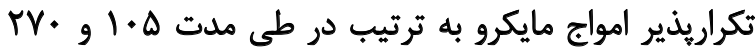

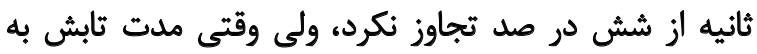

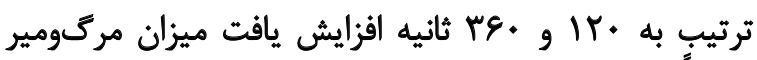

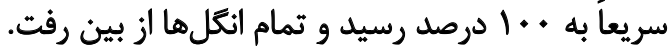

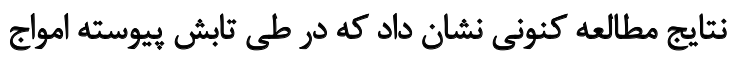

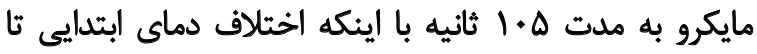

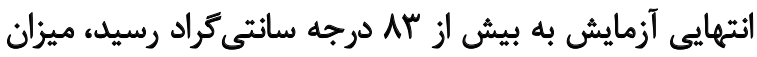

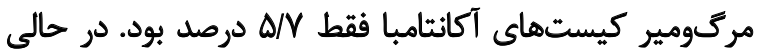

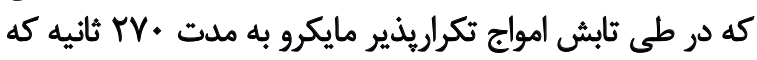

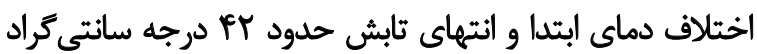

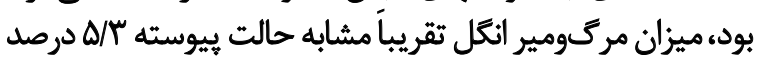

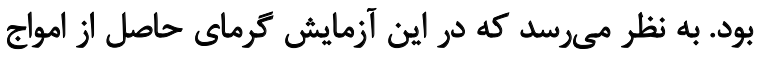

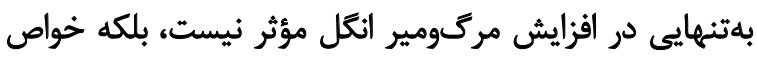

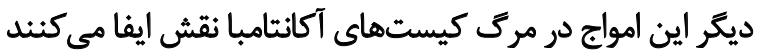

كه براى شناخت اين خواص نياز به مطالعات بيشترى است.

\section{تئيجهيَيرى}

نتايج اين مطالعه نشان داد كه با اينكه تغيير در شيوه تابش بأش

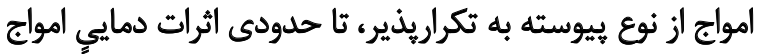

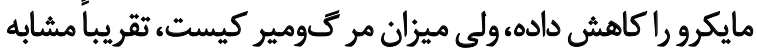

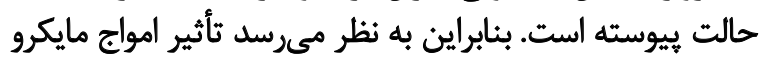

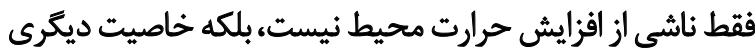

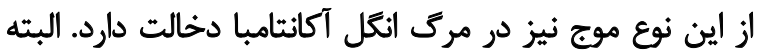

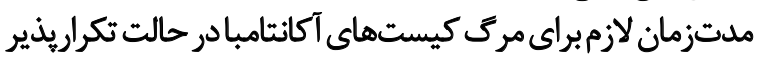

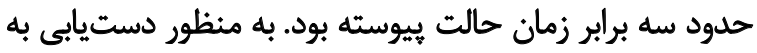

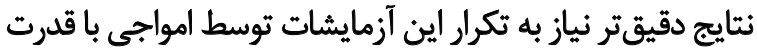

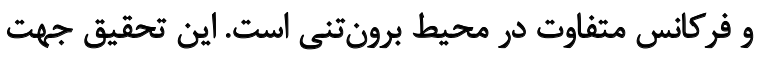

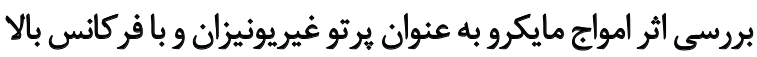

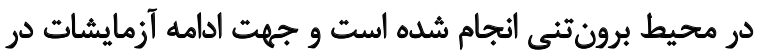

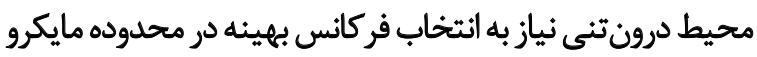

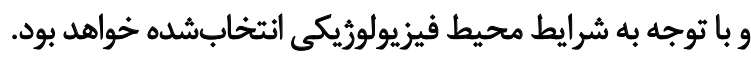

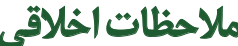 بيروى ازئ اصول الخاق يثوهش}

اين طرح يروهش با كد اخلاق 19-143-92 در كميته اخلاق دانشعاه علوميزشكى اراك به تصويب رسيده است.
اين انكل مىشود. به عبارت ديكر وجود كيستهاي آكانتامبا علت

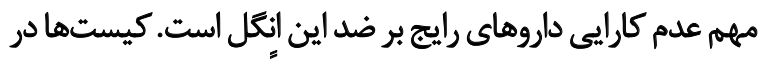

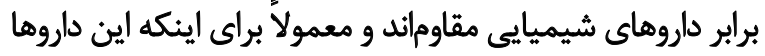

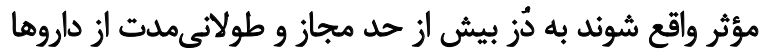

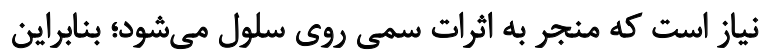

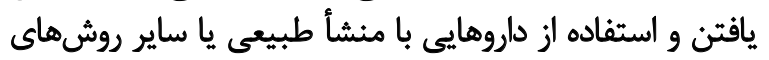

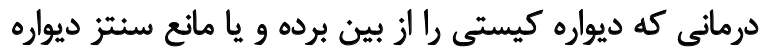

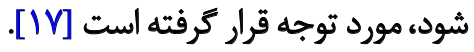

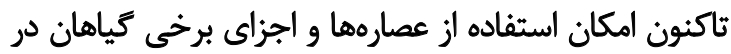

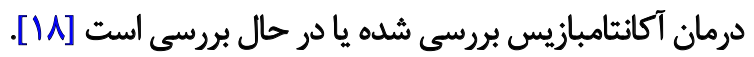
مصرف برخى از اين كياهان در طب سنتى رايج است؛ زيرا خواص

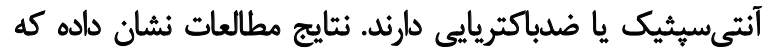

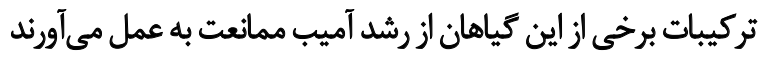

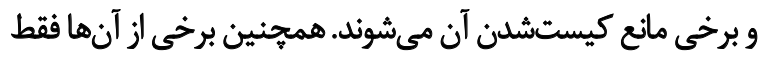

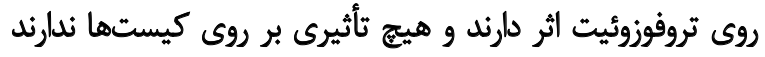

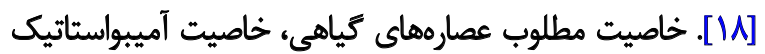

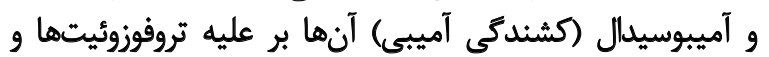

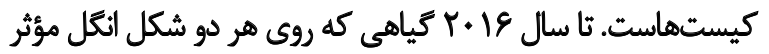

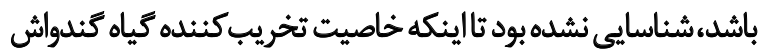

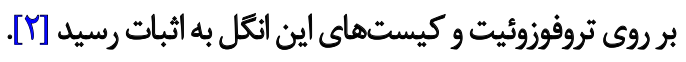

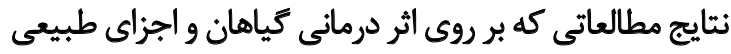

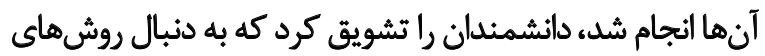

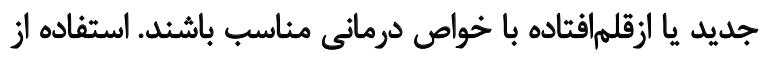

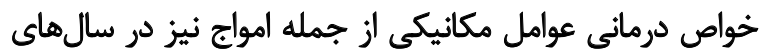
اخير مورد توجه قرار كرفته است

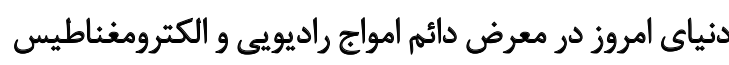

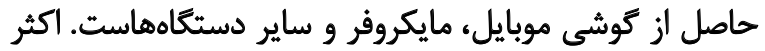

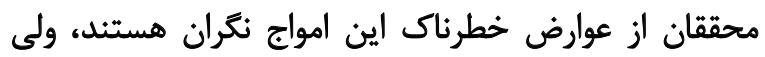

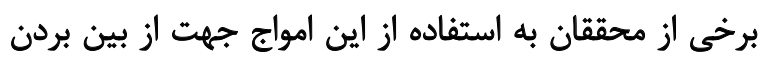

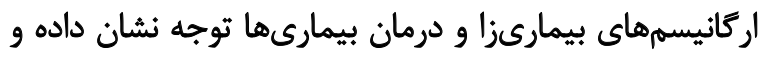

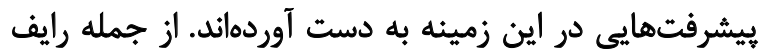

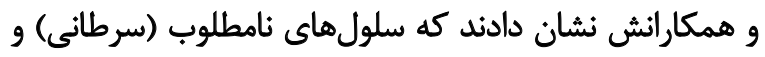

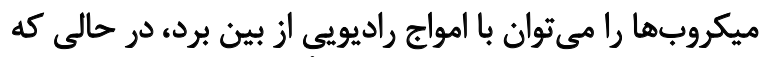

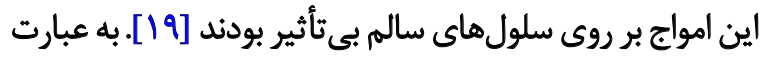

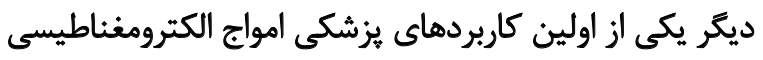

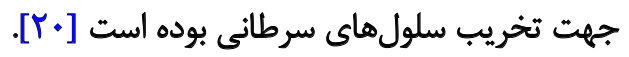

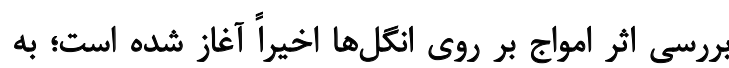

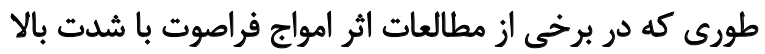

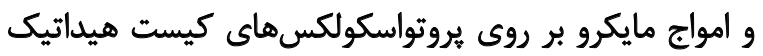

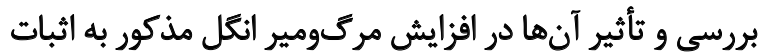

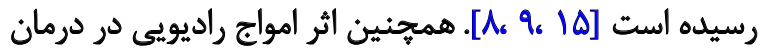

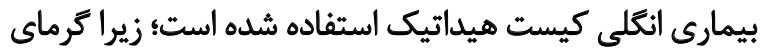




$$
\text { مامى مالى }
$$

اين برؤوهش باحمايت مالى معاونت تحقيقات وفناورى دانشعاه

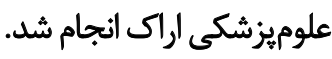

$$
\text { مشاركت نويسندكًان }
$$

نويسندكان به يك اندازه در نكارش يُوهش مشاركت داشتند.

$$
\text { تعارض مثافع }
$$

نويسندكان مقاله تصريح مي كنيند هيجگونه تضاد منافعى در

يثروهش حاضر وجود ندارد. 


\section{References}

[1] Niyyati M, Rezaeian M. Current status of Acanthamoeba in Iran: A narrative review article. Iranian Journal of Parasitology. 2015; 10(2):157-63. [PMID] [PMCID]

[2] Derda M, Hadaś E, Cholewiński M, Skrzypczak Ł, Grzondziel A, Wojtkowiak-Giera A. Artemisia annua L. as a plant with potential use in the treatment of acanthamoebiasis. Parasitology Research. 2016; 115:1635-9. [DOI:10.1007/s00436-016-4902-z] [PMID] [PMCID]

[3] Anwar A, Siddiqui R, Hameed A, Shah MR, Khan NA. Synthetic dihydropyridines as novel antiacanthamoebic agents. Medicinal Chemistry. 2019; 15:1. [DOI:10.2174/1573406415666190722113412] [PMID]

[4] Feiz Haddad MH, Shokri A, Habibpour H, Heidar Nejadi SM. A review of Acanthamoeba keratitis in the Middle East and Iran. Journal of Acute Disease. 2019; 8(4):133-41. [DOI:10.4103/2221-6189.263705]

[5] Müller GG, Kara-José N, de Castro RS. Antifungals in eye infections: Drugs and routes of administration. Revista Brasileira de Oftalmologia. 2013; 72(2):132-41. [DOI:10.1590/\$0034-72802013000200014]

[6] Siddiqui R, Aqeel Y, Khan NA. The development of drugs against acanthamoeba infections. Antimicrobial Agents and Chemotherapy. 2016; 60(11):6441-50. [DOI:10.1128/AAC.00686-16] [PMID] [PMCID]

[7] Hadaś E, Derda M, Cholewiński M. Evaluation of the effectiveness of tea tree oil in treatment of Acanthamoeba infection. Parasitology Research. 2017; 116(3):997-1001. [DOI:10.1007/s00436-017-5377-2] [PMID] [PMCID]

[8] Liu AB, Cai H, Ye B, Chen LL, Wang MY, Zhang J, et al. The damages of high intensity focused ultrasound to transplanted hydatid cysts in abdominal cavities of rabbits with aids of ultrasound contrast agent and superabsorbent polymer. Parasitology Research. 2013; 112(5):1865-75. [DOI:10.1007/s00436-013-3340-4] [PMID]

[9] Eslamirad Z, Soleimani H, Hajihossein R, Rafiei F. Evaluation of lethal effect of microwave exposure on protoscolices of hydatid cyst in vitro. Asian Pacific Journal of Tropical Disease. 2015; 5(10):821-4. [DOI:10.1016/S2222-1808(15)60938-0]

[10] Salmen SH, Alharbi SA, Faden AA, Wainwright M. Evaluation of effect of high frequency electromagnetic field on growth and antibiotic sensitivity of bacteria. Saudi Journal of Biological Sciences. 2018; 25(1):10510. [DOI:10.1016/j.sjbs.2017.07.006] [PMID] [PMCID]

[11] Ozdemir F, Kargi A. Electromagnetic waves and human health. In: Zhurbenko V, editor. Electromagnetic Waves. Rijeka: InTech; 2011. [DOI:10.5772/16343]

[12] Cairang Y, Zhang L, Ren B, Ren L, Hou L, Wang H, et al. Efficacy and safety of ultrasound-guided percutaneous microwave ablation for the treatment of hepatic alveolar echinococcosis: A preliminary study. Medicine. 2017; 96(27):e7137. [DOI:10.1097/MD.0000000000007137] [PMID] [PMCID]

[13] Meighani M, Eslamirad Z, Hajihossein R, Ahmadi A, Saki S. Isolation and genotyping of acanthamoeba from soil samples in Markazi Province, Iran. Open Access Macedonian Journal of Medical Sciences. 2018; 6(12):2290-4. [DOI:10.3889/oamjms.2018.454] [PMID] [PMCID]

[14] El-Sayed NM, Hikal WM. Several staining techniques to enhance the visibility of Acanthamoeba cysts. Parasitology Research. 2015; 114:82330. [DOI:10.1007/s00436-014-4190-4] [PMID]

[15] Eslamirad Z, Soleimani H. [Investigating the potential of protoscolices for cyst formation under in vivo microwave radiation (Persian)]. Complementary Medicine Journal. 2019; 9(1):3598-606. http://cmja.arakmu. ac.ir/article-1-647-en.html
[16] Mosayebi M, Hajihossein R, Ghorbanzadeh B, Kalantari S. A risk for nosocomial infection: contamination of hospital air cooling systems by Acanthamoeba spp. International Journal of Hospital Research. 2016; 5(1):17-21. [DOI:10.15171/ijhr.2016.04]

[17] Anwar A, Khan NA, Siddiqui R. Combating Acanthamoeba spp. cysts: What are the options? Parasites \& Vectors. 2018; 11:26. [DOI:10.1186/ s13071-017-2572-z] [PMID] [PMCID]

[18] Parasitology T, Ghaffarifar F, Fakhar M, Saberi R. Medicinal plants with anti-Acanthamoeba activity: A systematic review. Infectious Disorders - Drug Targets. 2019; 19:1. [DOI:10.2174/18715265196661907160958 49] [PMID]

[19] Atari Lar S, Ebrahimi M. [A reflection on the healing power of the waves (Persian)]. Journal of Vibration \& Sound. 2018; 7(13):104-19. http://jvs.isav.ir/article_32705.html

[20] Hedrick WR, Hykes DL, Strachman DE. Ultrasound physics and instrumentation. St. Louis: Mosby; 2005. https://books.google.com/ books?id=QoVsQgAACAAJ\&dq

[21] Botsa E, Thanou I, Nikas I, Thanos L. Treatment of hepatic hydatid cyst in a 7-year-old boy using a new type of radiofrequency ablation electrode. The American Journal of Case Reports. 2017; 18:953-8. [DOI:10.12659/AJCR.904432] [PMID] [PMCID]

[22] Du XL, Ma QJ, Wu T, Lu JG, Bao GQ, Chu YK. Treatment of hepatic cysts by B-ultrasound-guided radiofrequency ablation. Hepatobiliary \& Pancreatic Diseases International. 2007; 6(3):330-2. [PMID] 